

\section{DISCLAIMER}

This report was prepared as an account of work sponsored by an agency of the United States Government. Neither the United States Government nor any agency Thereof, nor any of their employees, makes any warranty, express or implied, or assumes any legal liability or responsibility for the accuracy, completeness, or usefulness of any information, apparatus, product, or process disclosed, or represents that its use would not infringe privately owned rights. Reference herein to any specific commercial product, process, or service by trade name, trademark, manufacturer, or otherwise does not necessarily constitute or imply its endorsement, recommendation, or favoring by the United States Government or any agency thereof. The views and opinions of authors expressed herein do not necessarily state or reflect those of the United States Government or any agency thereof. 


\section{DISCLAIMER}

Portions of this document may be illegible in electronic image products. Images are produced from the best available original document. 


\title{
SOFTWARE FOR A SPARK CHAMBER READER SYSTEM
}

\author{
S. Heller, J. Malament, L. Padwa, and M. Strongson
}

March 1966

\section{LEGAL NOTICE}

This report was prepared as an account of Government sponsored work. Neither the United States, nor the Commission, nor any person acting on behalf of the Commission:

A. Makes any warranty or representation, expressed or implied, with respect to the accuracy, completeness, or usefulness of the information contained in this report, or that the use of any information, apparatus, method, or process disclosed in this report may not infringe privately owned rights; or

B. Assumes any liabilities with respect to the use of, or for damages resulting from the se of any information, apparatus, method, or process disclosed in this report.

As used in the above, "person acting on behalf of the Commission" includes any employee or contractor of the Commission, or employee of such contractor, to the extent that ploye or contractor prepares, disseminates, or provides access to, any information pursuant to his employment or contract disseminates, or provides access to, any information pursuant
with the Commission, or his employment with such contractor.

\section{BROOKHAVEN NATIONAL LABORATORY UPTON, NEW YORK 11973}


This report was prepared as an account of Government sponsored work. Neither the United States, nor the Commission, nor any person acting on behalf of the Commission:

A. Makes any warranty or representation, expressed or implied, with respect to the accuracy, completeness, or usefulness of the information contained in this report, or that the use of any information, apparatus, method, or process disclosed in this report may not infringe privately owned rights; or

B. Assumes any liabilities with respect to the use of, or for damages resulting from the use of any information, apparatus, method, or process disclosed in this report.

As used in the above, "person acting on bchalf of thc Commission" includes any employee or contractor of the Commission, or employee of such contractor, to the extent that such employee or contractor of the Commission, or employee of such contractor prepares, disseminates, or provides access to, any information pursuant to his employment or contract with the Commission, or his employment with such contractor.

Printed in the United States of America

Available from

Clearinghouse for Federal Scientific and Technical Information National Bureau of Standards, U.S. Department of Commerce Springfield, Virginia 22151

Price: Printed Copy $\$ 3.00$; Microfiche $\$ 0.65$ 


\section{SOFTWARE FOR A SPARK CHAMBER READER SYSTEM}

\section{INTRODUCTION}

This report is concerned with a computer program whose aim is to recognize straight line patterns of sparks given a magnetic tape of input data produced by an off-line flying spot digitizer. ${ }^{1}$ The present film reading system uses the same digitizer and similar logical flow as the STEAM system. ${ }^{2}$ The programs described herein apply only to straight line tracks in rectangular chambers which may be arbitrarily positioned on film.

Wherever feasible the individual subprograms have been put in closed subroutine form. This facilitates handling and updating. It permits use of only part of the programming system when desired. The fiducial finding subroutine FIDUG is generalized to look for a single fiducial anywhere on the picture, with the area specified in the calling sequence. $\mathcal{N}$ fiducials are found with $\mathcal{N}$ calls, wherc $\mathcal{N} \geqslant 3$. The transformation to rectangular coordinates is done by series approximation rather than by table look-up. Although this takes slightly longer, it saves 2000 memory locations and increases accuracy. The transformation routine which merges two scans is applied as well to put all the data into the same coordinate system with respect to specified fiducials. Thus all pictures for the same experiment have the same frame of reference independent of the skew (rotation) or stop error ( $y$ translation) introduced by the film motion in the digitizer. The sORT subroutine processes one point at a time instead of making one pass over all the transformed data. It is thus called as many times as there are data points. This saves memory required to store the transformed points. The TRACK subroutine handles each view of each spark chamber separately, hence allowing for arbitrary configuration of chambers. A variety of input and assembly parameters chosen by the user determines the conditions for acceptance of data which form a spark, a track, or a useful event. A routine called veE to find v intersections of tracks and another called PRMGH which matches corresponding tracks in different views of the same chamber are included in the system. (However, the user is required to do his own spatial reconstruction.) The output is a magnetic tape and an optional printout which contains frame identification and a list of tracks with their respective sparks. The maximum information retrievable per spark is the first and last point digitized, the total number of points, and the average point (centroid). The centroids, which average out many digitizing errors, are used to compute the equation of a best fit line for the tracks. While the software system uses this equation, the user may recompute the equation using other points contained in the output so as to satisfy the user's statistical and kinematical analysis. Among the utility routines, a calibration routine is concerned with the digitizer itself. It uses FIDUC, an abbreviated version of the Main Program called PROG for transforming points, and a short FORTRAN control program VARY to analyze a test strip of grid film and thereby ascertain certain digitizer parameters.

The digitizer has undergone extensive examination and changes. An earlier report describes the overall capabilities of the entire film reading system with emphasis on the flying spot digitizer. ${ }^{3}$ It is sufficient to remark that the least counts have been halved (now an $x$ unit is 4.2 microns, a $y$ unit is 10 microns), and various monitoring features have been added. The net result is an improvement in accuracy and consistency. Maximum errors in digitization are of the order of 50 microns for a single point, with a maximum average error over any section of the frame of about 10 microns. Statistically, results tend to be better than these maxima.

These programs, which represent approximately four programmer years of effort, have been completely debugged and tested. Pictures such as those in Figures 1 and 2 have been successfully analyzed. 


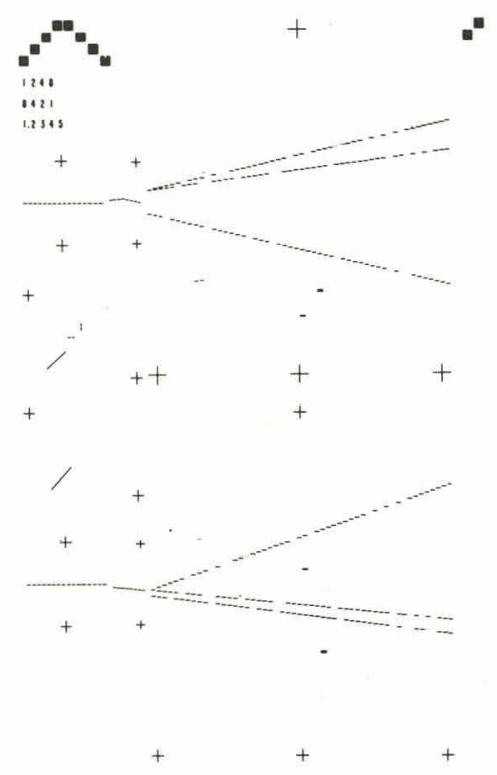

Figure 1. Picture of typical event actually processed, showing registration blocks and frame identification matrix.

Figure 2. CRT playback (mirror images of events in Figure 1) after digitization and rectangular transformations. Two scans are shown. The number 12345678 is fictitious, but it ordinarily corresponds to the binary coded decimal in the identification matrix.

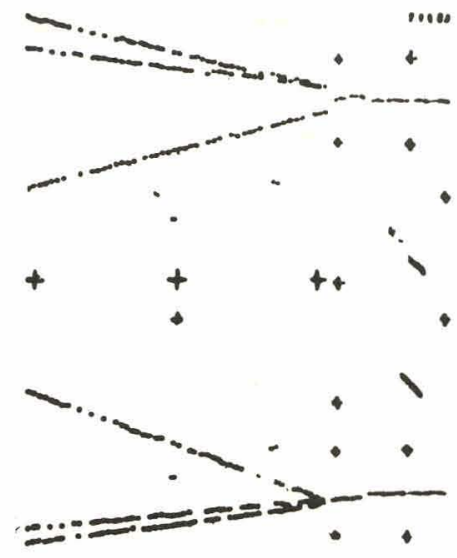

$1234 \quad 5673$
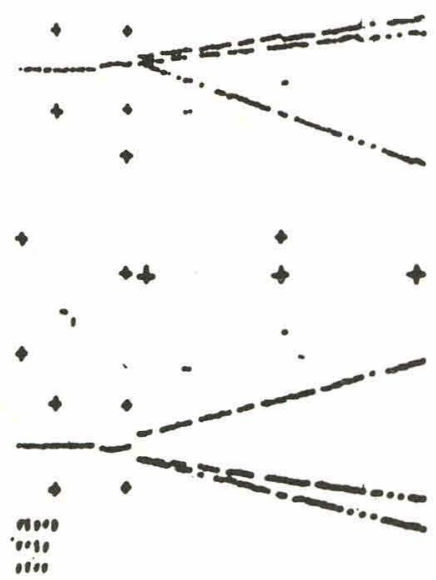


\section{USER REQUIREMENTS AND SYSTEM OPERTATIONS}

The proper use of an automatic system necessitates strict adherence to certain rules. This system has the following rules.

(a) High film quality, clean pictures, and good photography are essential for high efficiency.

(b) All fiducials should be plus-shaped cross marks, at least $1 \mathrm{~mm}$ in length and clear of all background by at least $0.5 \mathrm{~mm}$. At least 3 noncollinear fiducials are needed to perform transformations.

(c) When two scans are required (i.e., when sparks nearly parallel to both scans occur), the user should indicate which chambers should be assigned to each scan. This is input to the sORT routine.

(d) As presently set up, points closer together than $200 \mu$ may not be sensed, i.e., one point may not be digitized. Sparks thicker than $500 \mu$ will not be digitized. (See reference 3 for further details.) Selection of parameters should to some extent be guided by these limitations.

(e) A period of at least one to two weeks is needed to supply and test the correctness of various input and assembly parameters (details of the individual subprogram requirements and input parameters are indicated in the section on Programs) and to arrange the format of the output and labeling of tracks.

(f) Frame and reel number boxes and registration boxes should conform to the standard. ${ }^{3}$ The registration boxes enable accurate stopping of the film motion, and the frame and reel number boxes are sensed and recorded as binary coded decimal frame identifications.

(g) Spatial reconstruction and kinematical analyses are to be supplied by the user.

\section{Operating Instructions}

The complete spark chamber software package has 17 independently assembled routines (with common storage linkage) and one data card. The routines are listed in Figure 3, with the routines they call in parentheses.

The data card has 3 integer numbers ending in columns 5,10,15, respectively. The first represents the number of magnetic tapes to be processed, the second is 0 for no CRT pictures and nonzero for CRT pictures, and the third is 0 for no print-out (except for error print-outs) and nonzero for print-out of every event.

Routines $4,5,6,7,8,9,10,11,12,13,15$ should ordinarily not be reassembled regardless of the experiment. The others will initially have new or different assembly parameters and will generally have to be reassembled. The control cards will have a list of magnetic tapes in the order to be mounted on B6, and a list of output tapes to go on A6. The run deck is shown in Figure 4. Two subsystems that are not primarily concerned with pattern recognition are a calibration run deck and a fiducial run deck. The routines for the calibration are:
(1) VARY
(4) ONLN
(7) RDTP
(2) FIDUC
(5) BSTLN
(8) PROG
(3) COFS
(6) ERROR
(9) TRANSF

Routines with the same name as the larger run deck are not necessarily the same, i.e., variations required for the subsystem have been inserted.

The fiducial system is intended for finding fiducials only; it uses the following routines:
(1) PROG
(4) ONLN
(7) TRANSF
(2) FIDUC
(5) BSTLN
(8) FPRNT
(3) COFs
(6) ERROR

Only the XEQ control card (no data card) is required for either subsystem.

The main system and two subsystems are available as source decks or binary decks at the Applied Mathematics Department of Brookhaven National Laboratory. 
1 MAIN . (READ, JPLOT, SORT, ERROR, FIDUC, FPRNT, TRANSF)

2 . SORT (TRACK, ERROR)

3 TRACK (BSTLN, ERROR, PRMCH, MSG)

4 TRANSF

5 FIDUC (TRANSF, COFS, ONLN, BSTLN)

6 READ

7 FPRNT

8 . ERROR (RECRD1)

9 JPLOT

10 COFs

11 ONLN

12 BSTLN

13 MSC

14 PRMCH

15. VEE

16 FNOTPT

$17^{\circ}$ RECRD1

Figure 3.

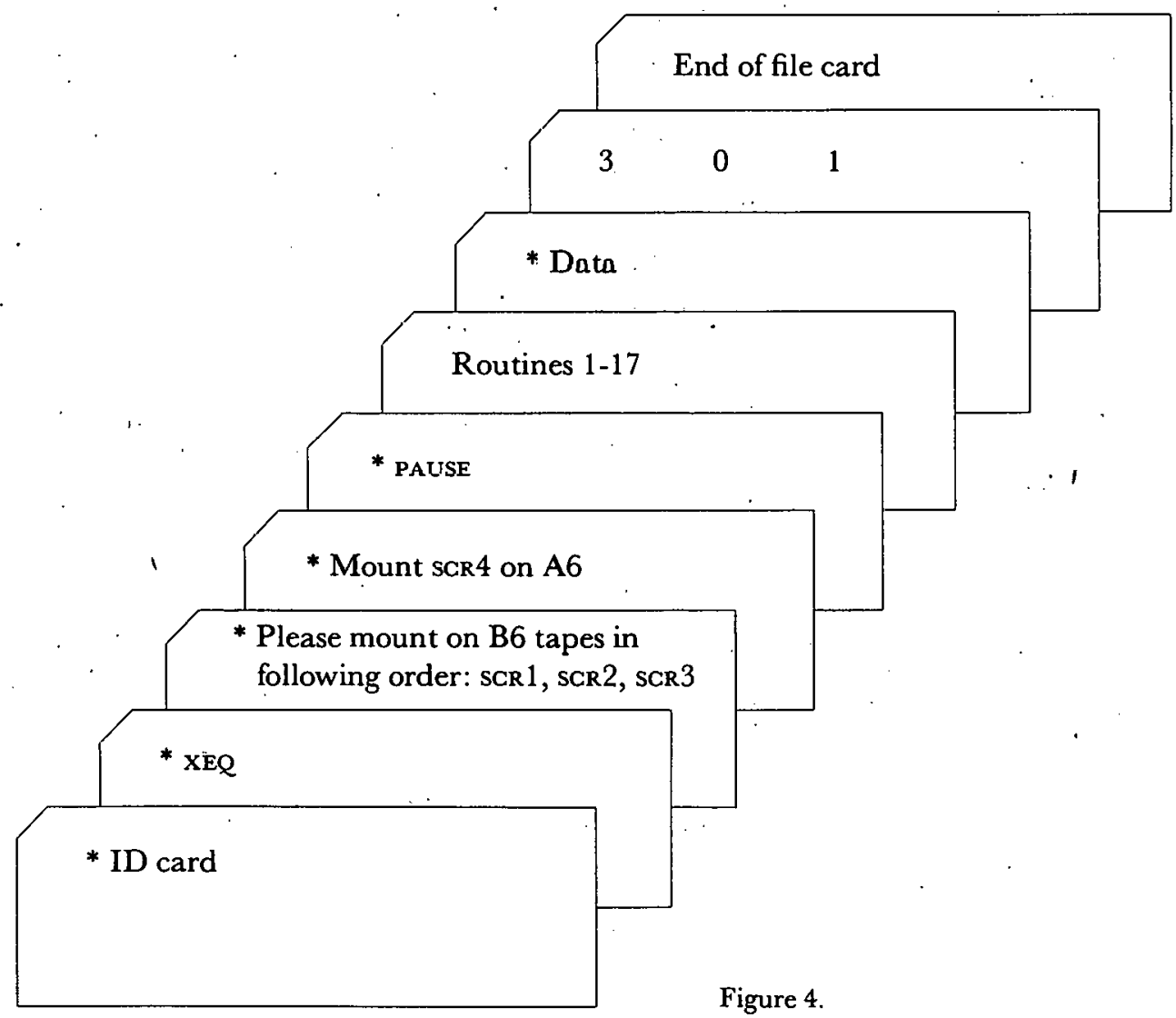


THE PROGRAMS

The logical flow of the program is indicated in Figure 5. This software package consists mainly of FAP subroutines to be run on the IBM 7094 computer. It time-shares with itself by reading in the next file while finishing up the computation and output on the present file. The total time to process one event having about 3000 digitizations is 1 second; the total length of the program is $25 \mathrm{~K}$ including buffers and working storage.
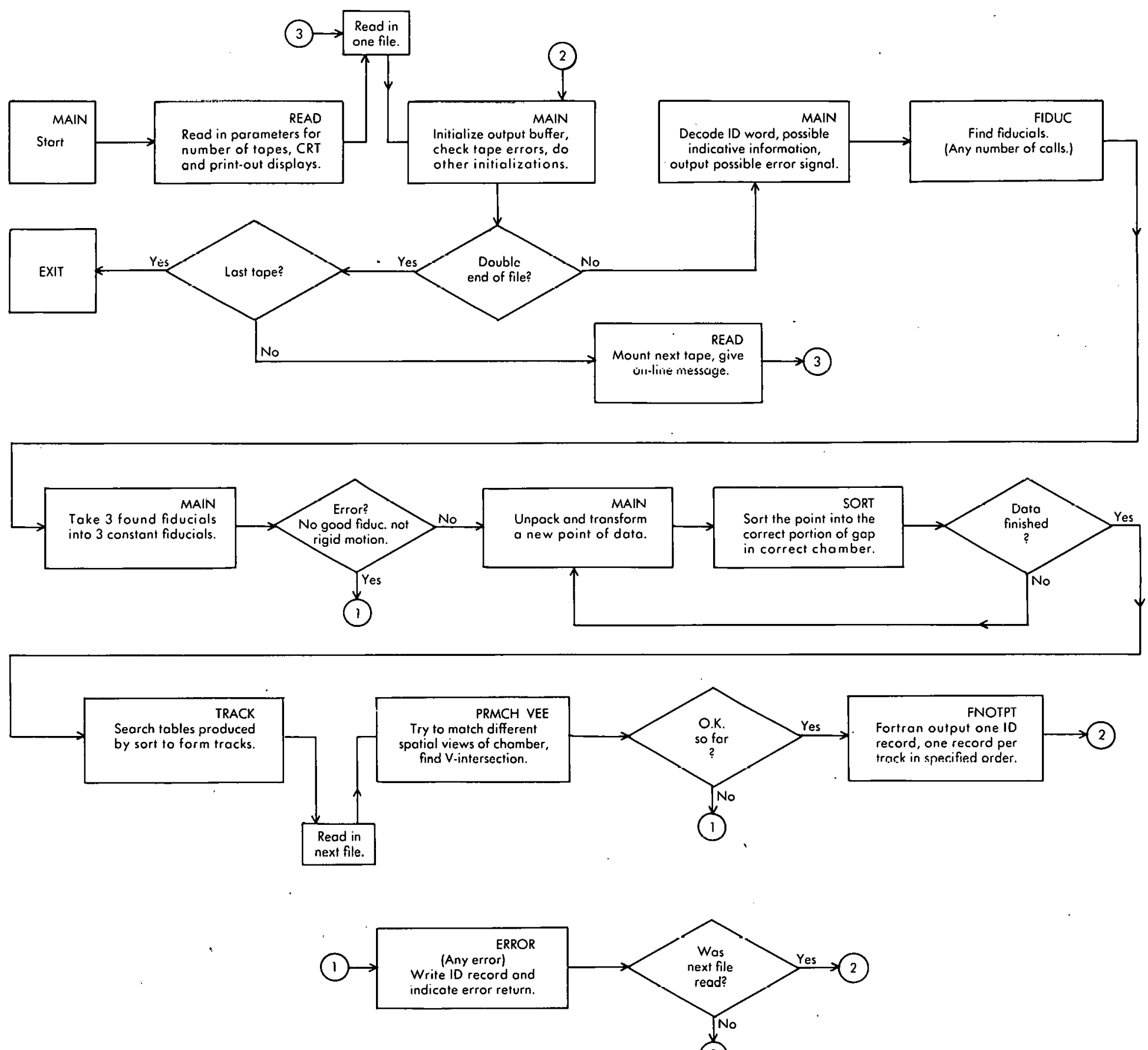

Figure 5. Program flow chart. 
The routines of central importance are given a full documentation while the shorter and less important ones are briefly described below. All FORTRAN refers to the FORTRAN II version.

READ: A FORTRAN routine which reads in control parameters at the beginning of execution, prints an operator message to mount next tape at the end of input tape, and calls EXIT at the end of the run.

FPRNT: For each frame, FPRNT prints the fiducials found in that frame. If a fiducial is not found, zeros are printed.

ERROR: If there is an error at any stage of the analysis, ERROR prints a message indicating the specific error.

JPLOT: A BNL system utility subroutine for plotting on the CRT and recording on film.

COFS: A short FORTRAN program to calculate quickly the equation of the straight line between two given points. COFs is much faster than BSTLN, but can only be used for two points.

ONLN: Given the equation of two lines and given a point, ONLN determines whether or not the point is on one of the lines within a prescribed tolerance.

FNOTPT: A FORTRAN program which writes the final output (sparks, tracks) on magnetic tape.

RECRD1: A FORTRAN program which writes one record on the nutput tape. This resord rnnsists of the roll and frame number of the event being processed together with possible error indication. RnTP: That part of the Main Program which reads the input tape. RDTP is used only in the calibration system.

PROG: That part of the Main Program which has the fiducial calling sequence and is used in the subsystems rather than in the main system.

\section{Name: Main Program}

Purpose: (a) To monitor control of subroutines.

(b) To read input tapes and data card.

(c) To unpack and transform data.

Method: The Main Program begins by calling READ which reads the data card giving the number of tapes and options on print-outs or CRT pictures. The first file of tape is read into memory and unpacked according to the format:

up to $r \geqslant 0$
indicative
words $\left\{\begin{array}{l}\text { first word } \quad \text { possibly indicative information, hit } 1 \text { contains a } 1 \\ \vdots \\ \text { up to } r \text { words of indicative information }\end{array}\right.$

one ID word \{Identification word: if bit 0 contains a 1 , then error for last (previous) file is indicated

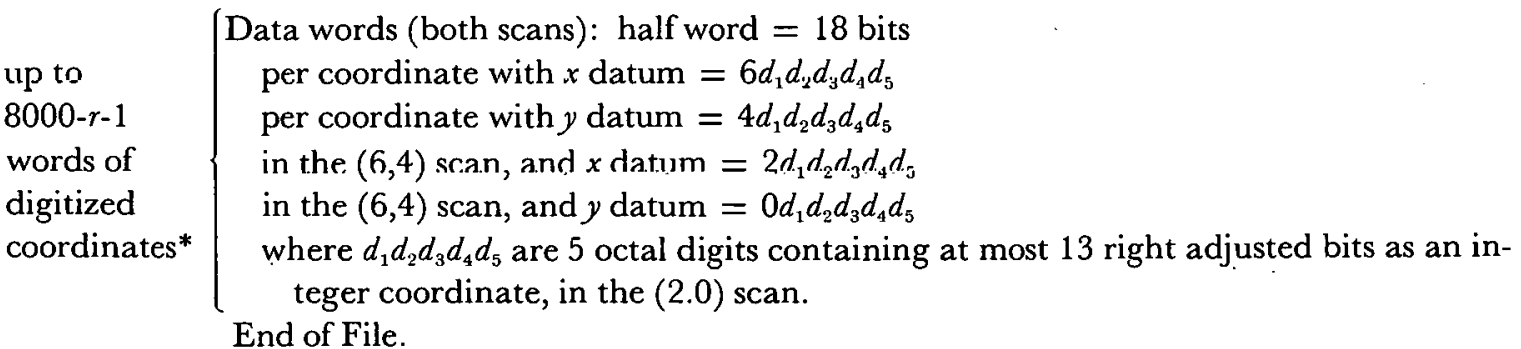

All the $x$ 's belonging to a $y$ scan line precede that $y$ coordinate. A scan may change in the middle of a word within the file. The file is composed of 64 word records with the last record filled out with

*The number 8000 may be changed if necessary. 
zeros. There may be no indicative data, in which case the identification word ( 4 binary coded decimal characters right adjusted in each half of the word) is the first word in the file.

The FIDUC subroutine is called to establish the fiducials which are to be mapped into three given ones. This mapping is determined by an open subroutine called Triduc. If only one scan will suffice for the entire picture, only one transformation is computed in TFiDuc. The scan for the picture is indicated by the value of the variable NSCAN which is 0,1 , or 2 for the $(2,0)$ scan, $(6,4)$ scan, or both scans, respectively (see page 12 for directions of different scans).

Each point is then converted from the film coordinates to rectangular coordinates by the routine TRANSF, and the rigid (or nearly rigid) motion determined by TFIDUC is applied. If a CRT plot is called for, the point is printed on the CRT. The sORT routine is called once for each datum. The beginning and end of data for the event are accompanied by transfer to the sORT entries, SORT 3 and SORT2, respectively. These are initialization and cleanup routines. SORT passes control on to TRACK, which initiates the read of the next file. If error conditions occur prior to TRACK, the next file is read in the Main Program. The output on magnetic tape occurs after TRACK. After all the files on all the tapes are processed, EXIT is called.

In the $(6,4)$ view, TRANSF transforms the film coordinates $\left(x_{l}, y_{l}\right)$ into rectangular coordinates $\left(x_{R}, y_{R}\right)$ by the formulas

$$
\begin{aligned}
& x_{R}=x_{f} \cdot \sin \alpha-(\cos \alpha) \cdot\left(R \cdot \cos \theta_{1}-M_{1}\right) \\
& y_{R}=y_{l}(2.3622)+x_{f} \cdot(\cos \alpha)\left(R \cos \theta_{1}-M_{1}\right)+(\theta j v / 2 \pi)+K_{1},
\end{aligned}
$$

where

$$
\begin{aligned}
\alpha & =44.943^{\circ}, R=37,500 x \text { units, } L=4000 x \text { units, } \\
M_{1} & =37286 x \text { units, } \cos \theta_{1}=\left[1-\left(L-x_{f} / R\right)^{2}\right]^{1 / 2} \approx 1-1 / 2\left(L-x_{f} / R\right)-1 / 8\left(L-x_{f} / R\right)^{4},
\end{aligned}
$$

$\theta p v / 2 \pi=$ the distance the stage has moved since the beginning of the scan,

$K_{1}=$ a translation factor to insure a positive integer result within a 15-bit range (presently set at minus $4000_{8}$ ), and

$2.3622=$ the ratio of $y$ to $x$ units $=10 / 4.2333$.

For the $(2,0)$ scan

$$
\begin{aligned}
& x_{R}=x_{1} \cdot \sin 45.057^{\circ}+\left(\cos 45.057^{\circ}\right)\left(R \cdot \cos \theta_{2}-M_{2}\right) \\
& y_{R}=y_{1} \cdot 2.3622-x_{1} \cdot \cos 45.057^{\circ}+\left(\sin 45.057^{\circ}\right)\left(R \cdot \cos \theta_{2}-M_{2}\right)+(\theta p v / 2 \pi)+K_{2},
\end{aligned}
$$

where $R=36,900 x$ units, $L=3420 x$ units, and $M_{2}=36,736 x$ units. The definition of $\cos \theta_{2}$ is the same as that of $\cos \theta_{1}$, and $K_{2}$ is presently set at $6000_{8}$.

Any point which transforms into a negative number is ignored or deleted as a candidate for the SORT analysis.

The TFIDUC routine takes three found fiducials $\left(x_{1}, y_{1}\right),\left(x_{2}, y_{2}\right),\left(x_{3}, y_{3}\right)$ into three fixed fiducials $\left(X_{1}, Y_{1}\right),\left(X_{2}, Y_{2}\right),\left(X_{3}, Y_{3}\right)$ to determine the rotation matrix

$$
\alpha=\left(\begin{array}{ll}
\alpha_{11} & \alpha_{12} \\
\alpha_{21} & \alpha_{22}
\end{array}\right)
$$

and the translation vector

$$
\beta=\left(\begin{array}{c}
\beta_{1} \\
\beta_{2}
\end{array}\right)
$$

according to the solutions of the equations 


$$
\begin{array}{ll}
X_{1}=\alpha_{11} x_{1}+\alpha_{12} y_{1}+\beta_{1} & \Upsilon_{1}=\alpha_{21} x_{1}+\alpha_{22} y_{1}+\beta_{2} \\
X_{2}=\alpha_{11} x_{2}+\alpha_{12} y_{2}+\beta_{1} & \Upsilon_{2}=\alpha_{21} x_{2}+\alpha_{22} y_{2}+\beta_{2} \\
X_{3}=\alpha_{11} x_{3}+\alpha_{12} y_{3}+\beta_{1} & \Upsilon_{3}=\alpha_{21} x_{3}+\alpha_{22} y_{3}+\beta_{2} .
\end{array}
$$

The fixed fiducials must be noncollinear to avoid singularities in the solutions for $\alpha$ and $\beta$. The program requires $|\alpha|=1$ to within a prescribed tolerance, i.e., the motion must be nearly rigid. To transform a point $\left(x_{R}, y_{R}\right)$ into a point in the fixed coordinate system, we have

$$
\left(\begin{array}{l}
x \\
y
\end{array}\right)=(\alpha) \cdot\left(\begin{array}{l}
x_{R} \\
y_{R}
\end{array}\right)+(\beta)
$$

Error Conditions: (1) Too many words in the file (the maximum is presently set at 8000 ).

(2) Scanner trouble preceding the present frame. An error message referring to the previous frame is printed out, but the analysis of the present frame is not affected.

(3) $y$ coordinate not monotonic as encountered in the file.

(4) Redundancy or parity errors on reading tape.

(5) Absolute value of determinant of rotation matrix $\alpha$ (as found in TFIDUc) not equal to 1.

(6) Result of division too large in computing $\alpha$ (singularity in the transformation).

(7) No end of file found in several attempts - next tape is processed.

Input to the Main Program: (a) The constant or fixed fiducials $\left(X_{i}, Y_{i}\right), i=1,2,3$ are put in the following locations prior to assembly time: CONFX1, CONFX2, CONFX3, CONFY1, CONFY2, CONFY3 as right adjusted integers representing $X_{1}, X_{2}, X_{3}, \Upsilon_{1}, \Upsilon_{2}, \Upsilon_{3}$. Copies of the above 6 viariables are put into CONFAl, CONFA2, CONFA3, CONFB1, CONFB2, CONFB3 as well.

(b) The calling sequence to the FIDUc subroutine (see FIDUC write-up) consists of input parameters prior to assembly time.

(c) The variable NSCAN is set prior to assembly time, with the value 0 for only the $(2,0)$ scan of the entire picture; the value 1 for only the $(6,4)$ scan of the entire picture; the value 2 for both scans of the entire picture.

(d) The CRT scaling is presently fixed for a picture which measures $24 \mathrm{~mm}$ vertically by $45 \mathrm{~mm}$ horizontally. For different size pictures, especially larger ones, the parameters $X_{\text {MIN }}, X_{\text {MAX }}, \Upsilon_{\text {MIN }}$,

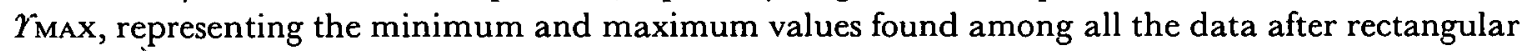
transformation, should be appropriately changed.

Time: With TRANSF and TFIDUC, approximately $150 \mu \mathrm{sec}$ per point.

Date: August 1965.

Authors: S. Heller, J. Malament.

\section{Name: FIDUC}

Purpose: 'To find a fiducial center.

Method: A binary search is performed on the packed data until a point is located within the scanner $y$ coordinate range for the fiducial ( $x$ and $y$ ranges are given in the calling sequence). After a one-by-one data count to get to the fringe of the $y$ range, all points in the eligible $x y$ region are examined for the geometric properties of a plus-shaped cross. Scan lines cut each of two pair of L-shaped legs at approximately $45^{\circ}$. All points such that the $x$ differences on successive $y$-scan lines monotonically decrease (until the center of the fiducial), then monotonically increase, are put in an array called FID and are transformed to rectangular coordinates by the TRANSF routine (q.v.). All $x$ differences are required to be less than the parameter XDIST (presently set at $800 \mu$ ). 
The approximate equation for the two cross lines $l_{1}$ and $l_{2}$ is determined by the condition that $l_{1}$ and $l_{2}$ intersect at $90^{\circ} \pm 10^{\circ}$. A transfer is made to a FORTRAN program ONLN in which points of the FID array are sorted on the lines if they fall within 8 units $(34 \mu)$ of $l_{1}$ or $l_{2}$. The points assigned on $l_{1}$ and $l_{2}$ are then fit to straight lines using the BSTLN routine (q.v.). The intersection of the two best lines is stored as the fiducial center.

Calling Sequence: FIDUc is called by the Main Program with the calling sequence:

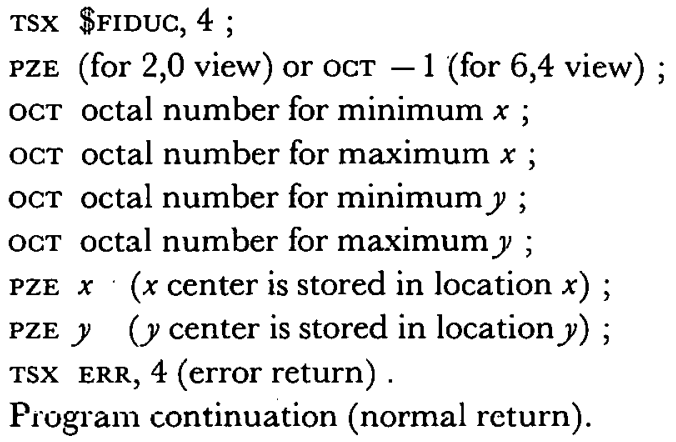

Error Refurns: If for any reasons the fiducials cannot be located, return is made by TRA 8,4 and zeros are stored for the fiducial center $(x, y)$.

Requirements: (1) Fiducials must be free of spark chamber data by at least $0.5 \mathrm{~mm}$ on film. (2) The total number of words in the file is in index register 2 on entry to Fiduc. (3) The fiducial area is initially determined by inspecting a binary dump of a typical event. A tolerance of \pm 0.5 in the $y$ direction (horizontal), and $\pm 0.1 \mathrm{~mm}$ in the $x$ direction is usually added to the actual size of the fiducial.

Length: 806 locations, approximately 500 executable.

Time: Approximately $20 \mathrm{msec}$ per fiducial.

Date: August 1965.

Authors: S. Heller, L. Padwa.

Name: BSTLN

Purpose: To compute, entirely in fixed point Fap, the "best" line (in a least squares sense) through a given set of points.

Use: The calling sequence to BSTLN must contain four arguments. The first argument must have, in its address, the location of an array of points and a tag of 1 . The next three arguments will have, in their addresses, the locations where the three coefficients of the line are to be stored. The calling program must also contain an array of points to be fitted to the line. In this array, each word will represent one point with $x$ in the decrement and $y$ in the address. Immediately preceding this array, there must be one word whose address contains the number of points in the array. It is the location of this word that is put into the address of the first argument of the calling sequence. The ouput from BSTLN consists of three numbers $(a, b$, and $c)$ which are the coefficients of the best line through the given points $(a x+b y+c=0)$. The three numbers produced will represent 35-bit fractions whose binary point precedes bit 1 . Also, the greater of $a$ and $b$ will be set equal to

$$
377777777777_{8}
$$

(which is the greatest possible coefficient) and the other two coefficients will be appropriately scaled.

Example: Suppose it is desired to fit a line through the following six points (all numbers octal):

$$
\begin{aligned}
& (2101,77777),(2200,77771),(2500,77740), \\
& (2440,77733),(2303,77720),(2000,77700) .
\end{aligned}
$$



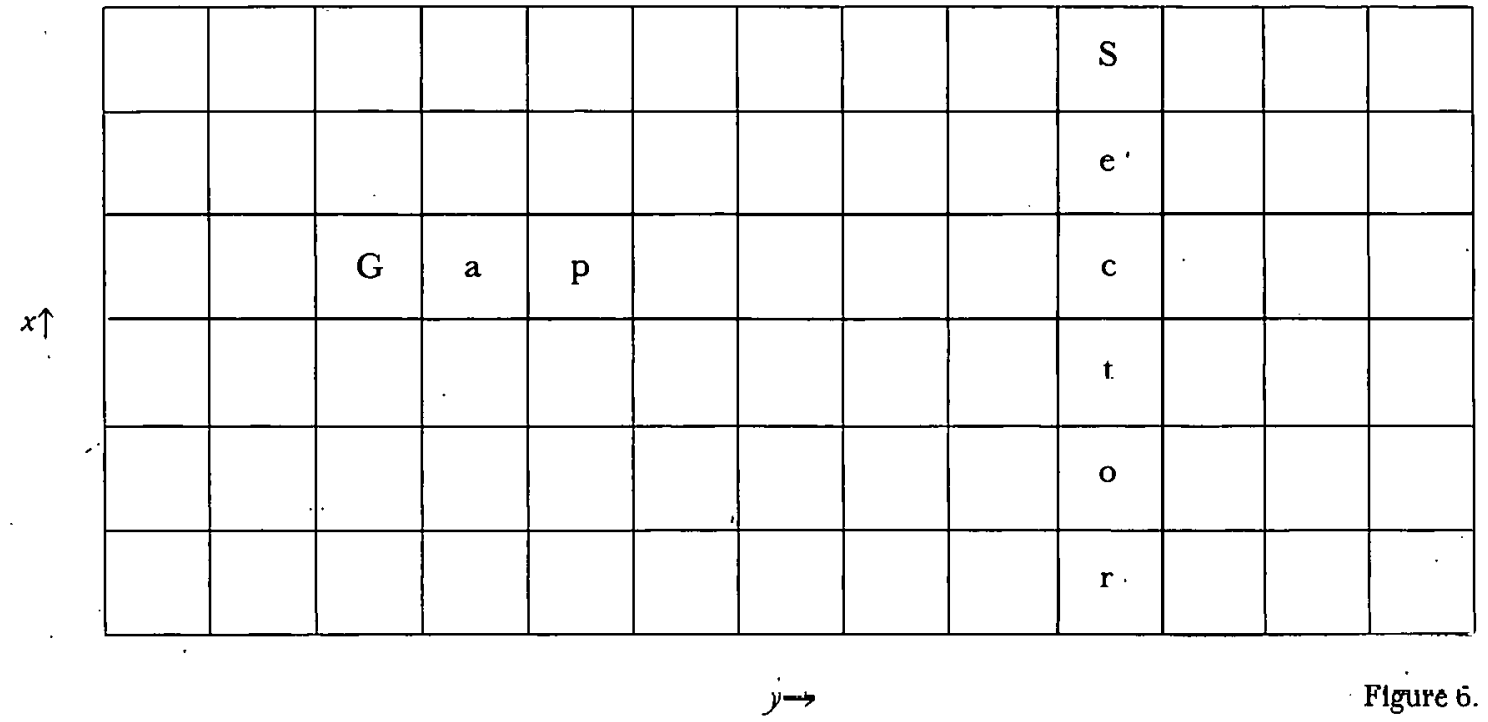

Figure 6.

Then the array of points would look like this:
ост 6
OCT 2101077777
UCI 2200077771
ocr 2500077740
OCT 2440077733
OCT 2303077720
OCT 2000077700

The calling sequence would be:

$$
\begin{aligned}
& \text { TSX } \text { SBSTLN, } 4 \\
& \text { PZE PNTS, } 1 \\
& \text { PZE } A \\
& \text { PZE } B \\
& \text { PZE } L^{\prime}
\end{aligned}
$$

After execution, the three locations with symbolic names $A, B$, and $C$ would contain the following:

$$
A=-000405527301, B=377777777777, C=-000000077736 \text {. }
$$

BSTLN returns to the location following the last argument in the calling program, i.e., IRA 5,4.

Method: Suppose there are $n$ points. First a line of the form $r x+s y+t$ is gotten with no restrictions on the magnitudes of $r, s$, and $t$. This is done as follows. Tet

$$
m=\left|\frac{y_{n}-y_{1}}{x_{n}-x_{1}}\right|
$$

$$
\begin{aligned}
& \text { If } m \leqslant 1, r=n \Sigma x y-\Sigma x \Sigma y, s=(\Sigma x)^{2}-n \Sigma x^{2} . \\
& \text { If } m>1, r=(\Sigma y)^{2}-n \Sigma y^{2}, s=n \Sigma x y-\Sigma x \Sigma y .
\end{aligned}
$$

Care must be taken in the calculation of these sums and products that no information is lost because of a number exceeding the fixed point limitations of a word. This is done by keeping an extra "overflow" word with each term whenever necessary, and increasing the overflow word each time the term exceeds the limits of a fixed point word. The terms are then appropriately shifted so that all terms have equal overflows. It is because of this feature that the routine can maintain thirty-five-bit ac- 
curacy without limiting the number of input points. Next, $r$ and $s$ are compared. If $|r|>|s|$, $x+(s / r) y+t / r=0$. But since $\bar{x}, \bar{y}$ is on the line, we have $\bar{x}+(s / r) \bar{y}+t / r=0$. Thus

$$
a=1\left(377777777777_{8}\right), \quad b=\mathrm{s} / \mathrm{r}, \text { and } c=-\bar{x}-\bar{y} ;
$$

If $r=s, a=b=1, c=-\bar{x}-\bar{y}$; and finally if $r=-s, a=-1, b=1, c=\bar{x}-\bar{y}$.

Restriction: Each $x$ and $y$ input coordinate must be a non-negative integer $\leqslant 77777_{8}$. There is virtually no restriction on the number of input points.

Length: 278 words.

Time: Approximately $500 \mu \mathrm{sec}+20 \mu \mathrm{sec}$ per point.

Date: March 1965.

Authors: S. Heller, L. Padwa.

\section{Name: SORT}

Purpose: (1) For each transformed datum point:

(a) To determine whether or not the point is in a chamber, and if it is, to determine which chamber.

(b) To transform the point from "film rectangular coordinates" (one rectangular system for the entire picture) to "chamber rectangular coordinates" ( a separate rectangular system for each chamber).

(c) Once in chamber coordinates, to check that the point is actually in a gap and not on one of the metal plates.

(d) To determine the sector and gap to which the point belongs (see Figure 6). The sector is a function of the $y$ coordinate, and the gap is a function of the $x$ coordinate.

(2) To list those sectors and gaps which contain points; to order the entries in the list by increasing gap number, and within onc gap by increasing sector number; to calculate the number of points, centroid, first, and last points of each sector.

SORT has three entry points - SORT3, SORT2, and SORT. SORT 3 is called once at the beginning of each event to zero arrays and initialize parameters. SORT is called once for each point. sORT puts the points in sectors and gaps and lists the sectors. SORT2 is called once for each event after all points have been put into sectors and gaps. SORT2 calculates centroids and calls TRACK.

Preliminory Setup: (1) Coefficient array: For each rectangular chamber, twelve words must be in this array. These twelve words are the coefficients of the lines making up the sides of the rectangle (threc for each side of the rectangle or twelve for the entire chamber). The three coefficients for each side, $a, b, c$, where $a x+b y+c=0$, must be normalized - i.e., $a^{2}+b^{2}=1$. Also, all coefficients must be in fixed point, and the binary point must be in the same place for all coefficients in all chambers.

The best place to put the binary point can be found by taking the maximum distance from any side to the origin and noting how many integer places this maximum distance requires (since in the normalized equation of the line, this distance is precisely the ahsolute value of the constant term). For example, suppose the maximum distance from any chamber to the origin is 80 units. The number 80 requires seven bits, so the binary point for all coefficients should follow bit 7 . This will give 28 bits of fractional accuracy. However, it has been found unnecessary to carry more than 15 to 18 bits of fractional accuracy, su lhe biinary point can be put after bit 8 instear of after bit 7 , losing one bit of fractional accuracy (the fraction will now be correct to 27 bits - still more than adequate). The motivation behind putting the binary point after bit 8 rather than bit 7 is that when this is done, the octal number thus formed can be read by inspection without doubling or halving. This will be true whenever the binary point is after bit 2 , or 5 , or 8 , or 11 , etc. This, of course, is not essential, but is merely a matter of convenience. 
The order of the entries in the coefficient array is important. The first twelve words refer to chamber 1 , the next twelve refer to chamber 2 , etc. Within the twelve words for one chamber, the first three coefficients are for the line parallel to the plates on the side with low gap numbers, and the remaining sides follow in counterclockwise order. (See Figure 7.) In each set of three coefficients, the first is the coefficient of $x$ (denoted as cox), the second is the coefficient of $y$ (coY), and the third is the constant term (denoted con). Thus the entire array appears in memory as shown in Figure 8, where the first subscript is the chamber number and the second subscript is the line number (i.e., which of the four sides of the chamber is being used).

(2) $\mathcal{N}$ array: For each chamber there will be one word containing thrce pieces of information. The sign bit tells which scan is correct for this chamber ( 0 for 2,$0 ; 1$ for 6,4$)$. The decrement (3-17) will have the number of gaps. The address (21-35) will have the sector width (denoted SCTwTH). A good first guess for the sector width is the average gap width. However, if it is found that this value does not produce the best results, it may be changed. All the sector widths (i.e., for all the chambers) are 15-bit fixed-point numbers, and they all must have the binary point in the same place. Where to put the binary point is resolved by putting it as far to the left as possible while still leaving enough room to the left of the binary point to put in the biggest integer needed. For example, if the biggest of all the sector widths is 28 , then the binary point would go after the fifth bit of the address (i.e., after bit 25 of the 36-bit word), allowing for five-bit integers and ten-bit fractions. It is also highly desirable for the sector width to be an exact divisor of the chamber width so that all sectors in one chamber will be equal. (See Figure 9.)

Finally the $\mathcal{N}$ array has a heading word, $\mathcal{N}$, which contains the number of chambers. Thus the entire array appears in memory as shown in Figure 10.

Line 1: Coefficients 1, 2, 3

Line 2: Coefficients 4, 5, 6

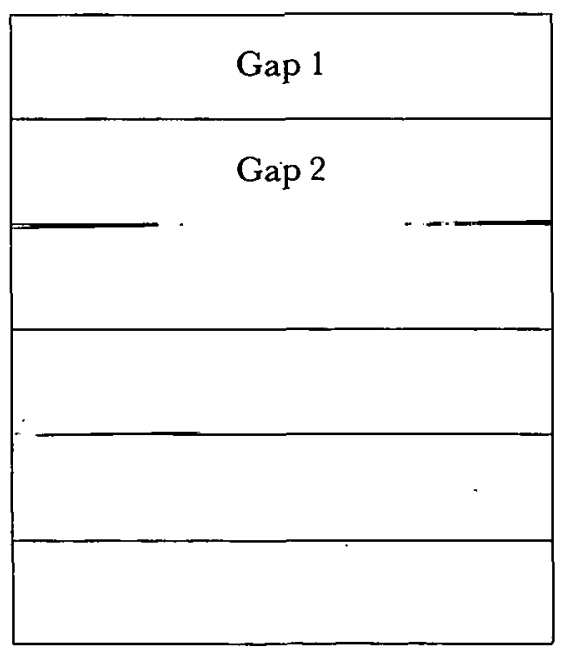

Line 3: Coefficients 7, 8, 9

Figure 7.
COEFF

Line 4: Coefficients 10, 11, 12

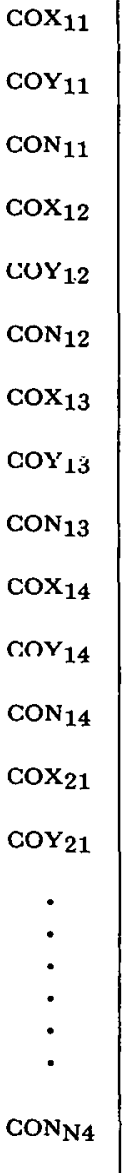


(3) ALPHA array: This is where the sectors containing points will be listed. It is therefore essential that the array be large enough to accommodate all the sectors which have points. Thus an upper bound on the number of sectors which contain points must be decided on. The array must also be able to accommodate one extra word per chamber. If at any time there are more sectors with points than the upper bound decided upon, the program will terminate the current frame and indicate that there are too many sectors with points. For example, if there are 8 chambers and it is desired to allow a total (i.e., in all chambers) of 800 sectors with points, then at assembly time the array must be written as follows:

\section{ALPHA $\quad$ BSS 808}

(800 sectors with points plus one word per chamber).

(4) BETA array: This array must be exactly four times as long as the maximum number of sectors with points allowed. Thus in the previous example of 8 chambers and 800 allowable sectors, one would write

BETA BSS 3200

NotE: In figuring the length of BETA, one does not use the extra word per chamber as in ALPHA. That is, to get the length of BETA, we took $4 \times 800=3200$, not $4 \times 808$.

(5) GAP array: This array contains, in scaled (see below) chamber coordinates, the first and last $x$ of each gap. It is possible, though not required, for the last $x$ of one gap to equal the first $x$ of the next gap. Any points that are found to be on a plate rather than between plates will be thrown out.

Scaling. All $x$ coordinates are $\leqslant 17777_{8}$ (i.e., 13 bits). Thus if the greatest $x$ coordinate in chamber coordinates takes up no more than 12 bits, each $x$ can be doubled and still be $\leqslant 17777_{8}$. The advantage in this is that one more bit of accuracy will be maintained. Similarly if the greatest $x$ takes up no more than $k$ bits $(k \leqslant 13)$, then all $x$ 's can be multiplied by $2^{13-k}$. Furthermore, the greatest $x$ in chamber coordinates is simply the $x_{\text {LAST }}$ value of the last gap of the longest chamber. Thus the scale value (i.e., the greatest power of two that the largest $x$ can be multiplied by and still be $\leqslant 17777_{8}$ ) is easily found from this particular $x$ value.

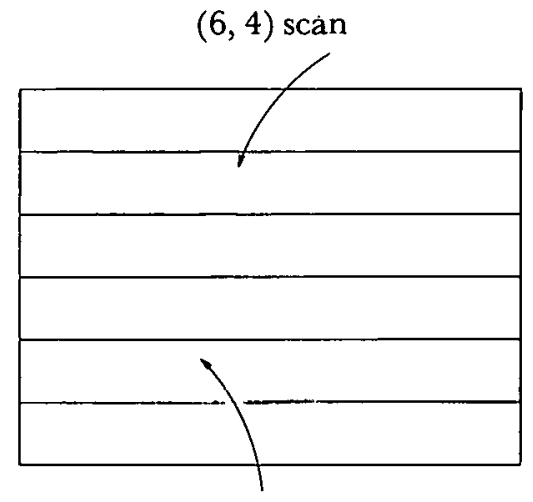

$(2,0) \operatorname{scan}$

Figure 9.

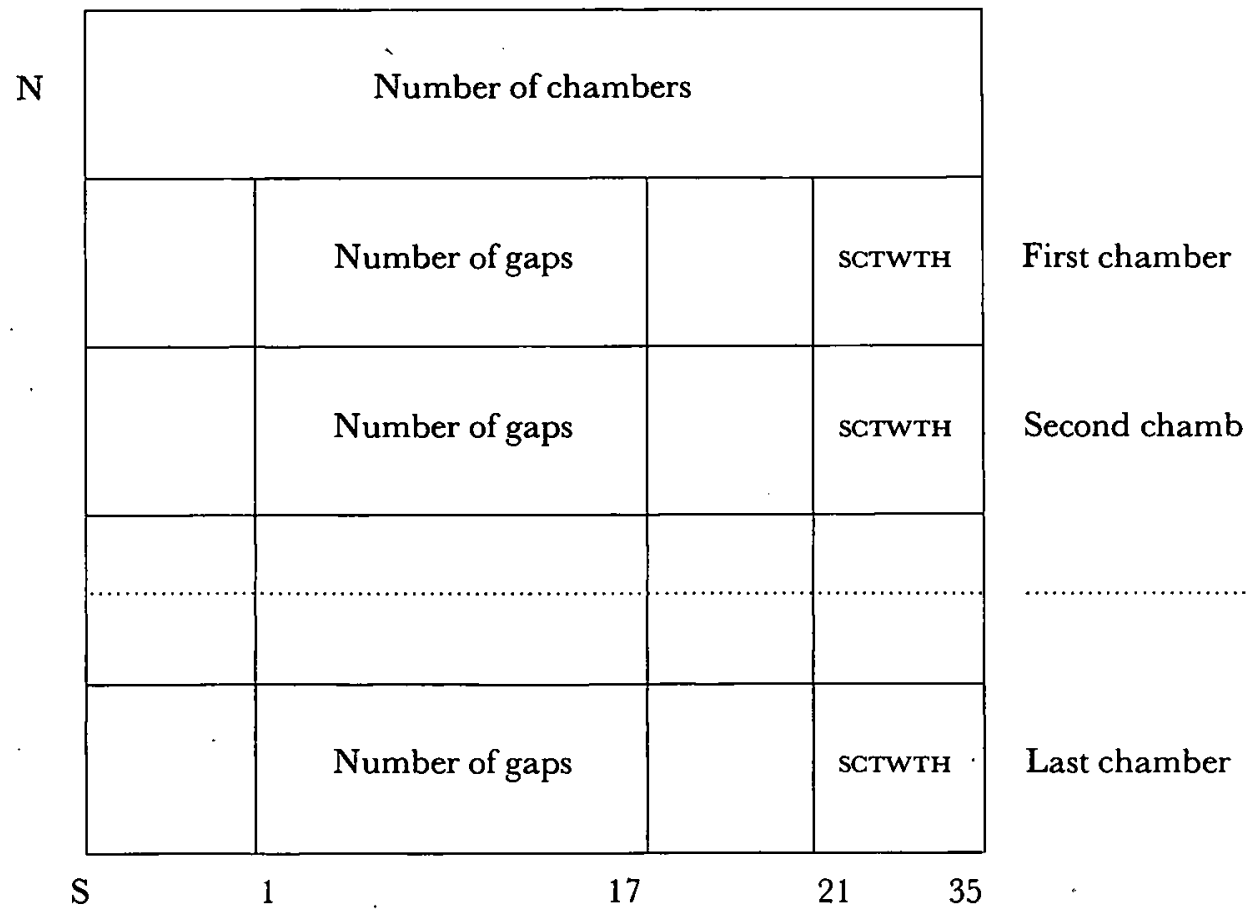

Sign bit, $S$, will have 0 if $(0,2)$ scan is correct for this chamber. Sign bit will have 1 if $(4,6)$ scan is correct for this chamber. 
Thus the scaled coordinates in the GAP array are 13-bit integers (i.e., binary point at far right). The scale value is always a power of 2 . It is generally either $1,2,4,8$, or 16 . It is a function of the length of the longest chamber.

The GAP array appears in memory as shown in Figure 11.

(6) Instructions to be altered. The instructions noted here must be altered for each experiment. In the listing, those instructions affected have asterisks and the change number in their comments.

***Change $1^{* * *}$. [Next to last instruction (ARS) of the macro VAL.] If the scale value (described above in discussion of GAP array) is $2^{k}$ and if the binary point in the coefficients is after bit $m$, then the address of this ARs instruction is $35-(k+m)$.

Example: If the scale value is $2^{1}$ and the binary point in the coefficients follows bit 14 , then this instruction should read ARS 20.

***Change $2 * * *$. Address must contain five times the maximum number of allowable sectors with points. For example, if 800 sectors with points are allowed, then this instruction reads AxT 4000,7 .

$\begin{array}{lllllll}\mathbf{S} & 4 & 3 & 1 \% & 18 & 22 & 23\end{array}$

Gap

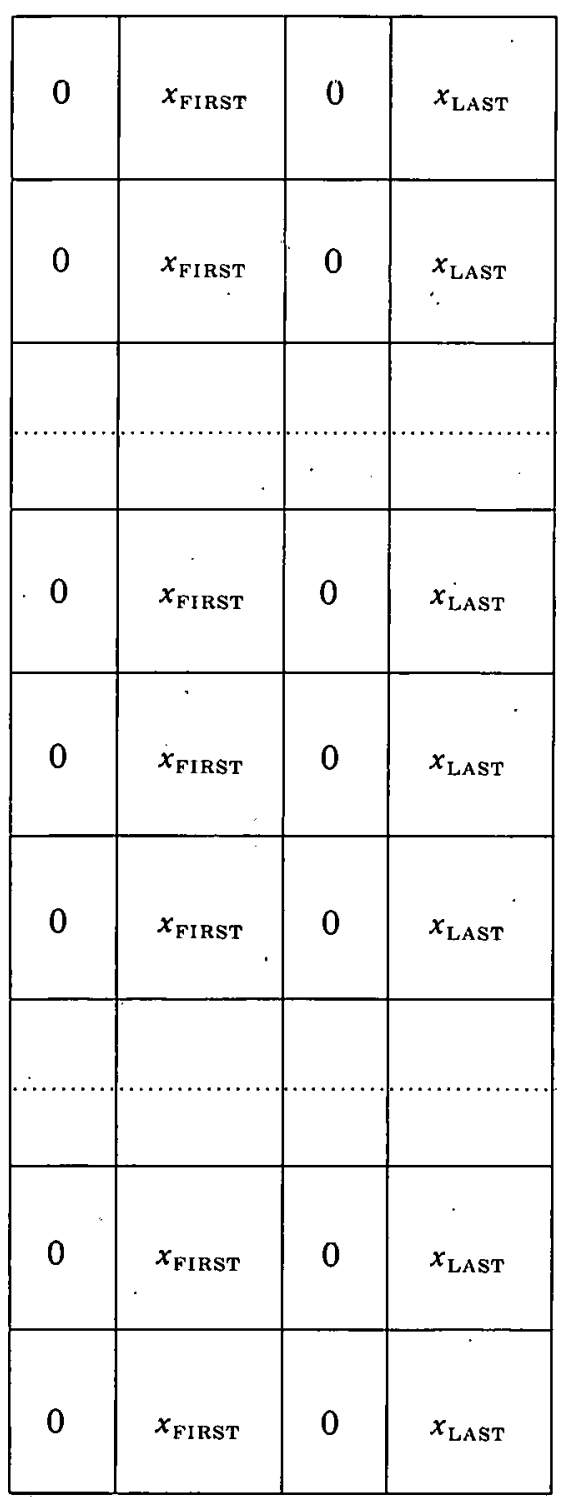

$\mathbf{S}$

$\begin{array}{llll}17 & 18 & 22 & 23\end{array}$
Fịrst gap, first chamber

Second gap, first chamber

Last gap, first chamber
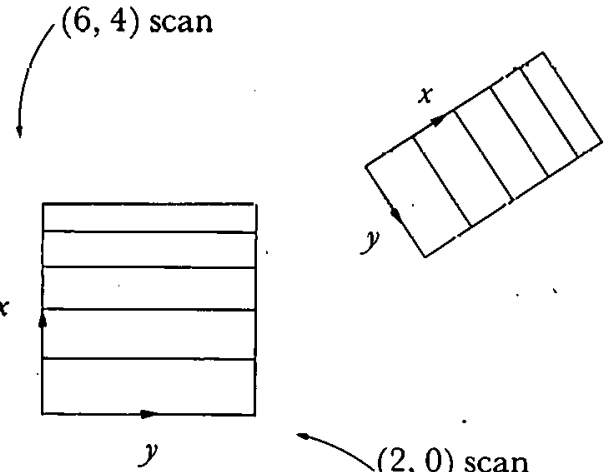

First gap, second chamber

$\left(x_{4}, y_{4}\right)^{\circ}$

$\left(x_{3}, y_{3}\right)$

Second gap, second chamber

Next to last gap, last chamber

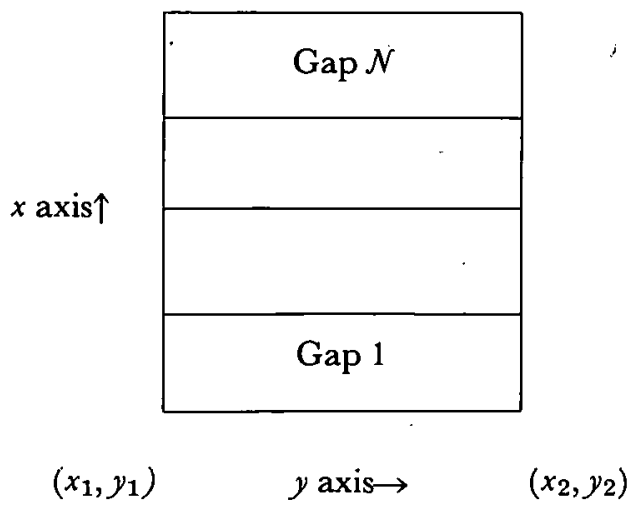

Last gap, last chamber

Figure 11. 
${ }^{* * *}$ Change $3^{* * *}$. If the scale value is $2^{k}$ and if the binary point in the sector widths is after bit $m$, then the address of this ALs instruction is $15-(k+m)$. For example, if the scale value is $2^{1}$ and the binary point in the sector widths is after bit 6 , then this instruction reads ALs 8 .

${ }^{* * *}$ Change $4^{* * *}$. The literal is the number of allowable sectors with points. For example, if 800 sectors with points are allowed, the instruction reads CAS $=800$.

(7) Calculation of coefficients and sector width. In each chamber, two of the sides of the chamber will be the axes for the chamber coordinate system. The $y$ axis will be parallel to the plates and the $x$ axis perpendicular to the plates. This is taken care of automatically by the program. However, in calculating the coefficients of the sides of each rectangle for the coefficient array, the $x$ axis must be horizontal and the $y$ axis vertical. Suppose we are given the coordinates of the four corners of a rectangular chamber, and we wish to calculate the coefficients of the sides of the chamber for the COEFF array (see Figures 12 and 13). In applying the following formulas, it is necessary to label the four corners precisely as in Figure 13. The plates must be parallel to the line joining $\left(x_{1}, y_{1}\right)$ and $\left(x_{2}, y_{2}\right)$. Also $\left(x_{1}, y_{1}\right)$ and $\left(x_{2}, y_{2}\right)$ must be along gap 1 . Thus in the chamber system $\left(x_{1}, y_{1}\right)\left(x_{2}, y_{2}\right)$ will be the $y$ axis, and $\left(x_{1}, y_{1}\right)\left(x_{4}, y_{4}\right)$ will be the $x$ axis. The origin will be at $\left(x_{1}, y_{1}\right)$. In the following formulas, $\operatorname{cox}_{j}, \operatorname{COY}_{j}, \operatorname{coN}$, are the coefficients of the line going from $x_{j} y_{j}$ to $x_{j+1} y_{j+1}$, where $\left(x_{5}, y_{5}\right)$ is the same as $x_{1}, y_{1}$.

(a) $\mathrm{DFN}_{j}=\sqrt{\left(x_{j}-x_{j+1}\right)^{2}+\left(y_{j}-y_{j+1}\right)^{2}} \quad \cdot j=1,4$

(b) $\operatorname{cox}_{j}=\frac{y_{j}-y_{j+1}}{\operatorname{DEN}_{j}}$

$$
\begin{aligned}
& \mathrm{COY}_{j}=\frac{x_{j 11}-x_{j}}{\operatorname{DEN}_{j}} \\
& \mathrm{CON}_{j}=\frac{x_{j} y_{j+1}-y_{j} x_{j+1}}{\mathrm{DEN}_{j}} \quad j=1,4
\end{aligned}
$$

(c) The leading coefficient must be positive. Thus if cox is negative, the signs of all three coefficients must be changed, and if $\operatorname{cox}=0$ and coY is negative, the signs of coy and con must be changed.

If it is desired to have the sector width equal to the average gap width and yet be an exact divisor of the chamber width, this may be done as follows. Let $m=$ integer closest to $\left(\mathrm{DEN}_{1} / \mathrm{DEN}_{2}\right) \times$ number of gaps. Then scTWTH $=\mathrm{DEN}_{1} / m$. There will be $m$ sectors. All these formulas must, of course, be applied independently to each chamber.

(8) Calling sequence and error returns. All three entry points are called by the Main Program.

(a) SORT 3 is called by TSX SSORT3,4. There are no arguments and no error returns. The normal return is TRA 1,4 .

(b) SORT is called by TSX $\$$ SORT,4. The AC must contain the coordinates of the transformed point to be sortcd ( $x$ decrement, $y$ address). Also, the sign bit of the AC must be on (negative) if the point came from the 6,4 view and it must be off (positive) if the point came from the 2,0 view. A fatal error (i.e., frame analysis termination) occurs if the current point starts a new sector, but there are no more allowable sectors with points. The error return is TRA 2,4 . The normal return is TRA 1,4 .

(c) SORT2 is called by TSx $\$$ SORT2,4. There are no arguments. A fatal error occurs if the total number of sectors with points is less than 2. If there is no error, SORT2 calls TRACK which proceeds further with the analysis. The error return from SORT2 is TRA 4,4. The normal return is TRA 1,4 .

Method: Since the equations of the sides of the chambers are in normal form, the directed distance from a point to a side is found by simply substituting the coordinate of the point into the equation. Also if the point is between two parallel lines, then the distances from the point to each line will have opposite signs. Thus if a point when substituted in the equations of opposite sides of a chamber results in the same sign, then the point is not in the chamber. 
Notation: In this flow chart a number enclosed in-a circle on any path logically corresponds to a place in the listing whose symbolic name is that number preceded by 'SRT. For example, $\rightarrow 11 \rightarrow$ corresponds to location SRT 11. $n$ is the total number of chambers. $(x, y)$ are the coordinates of the point input to SORT.

Length: Approximately 400 executable instructions.

Time: Approximately $150 \mu$ sec per point.

Date: February 1965.

Author: L. Padwa.

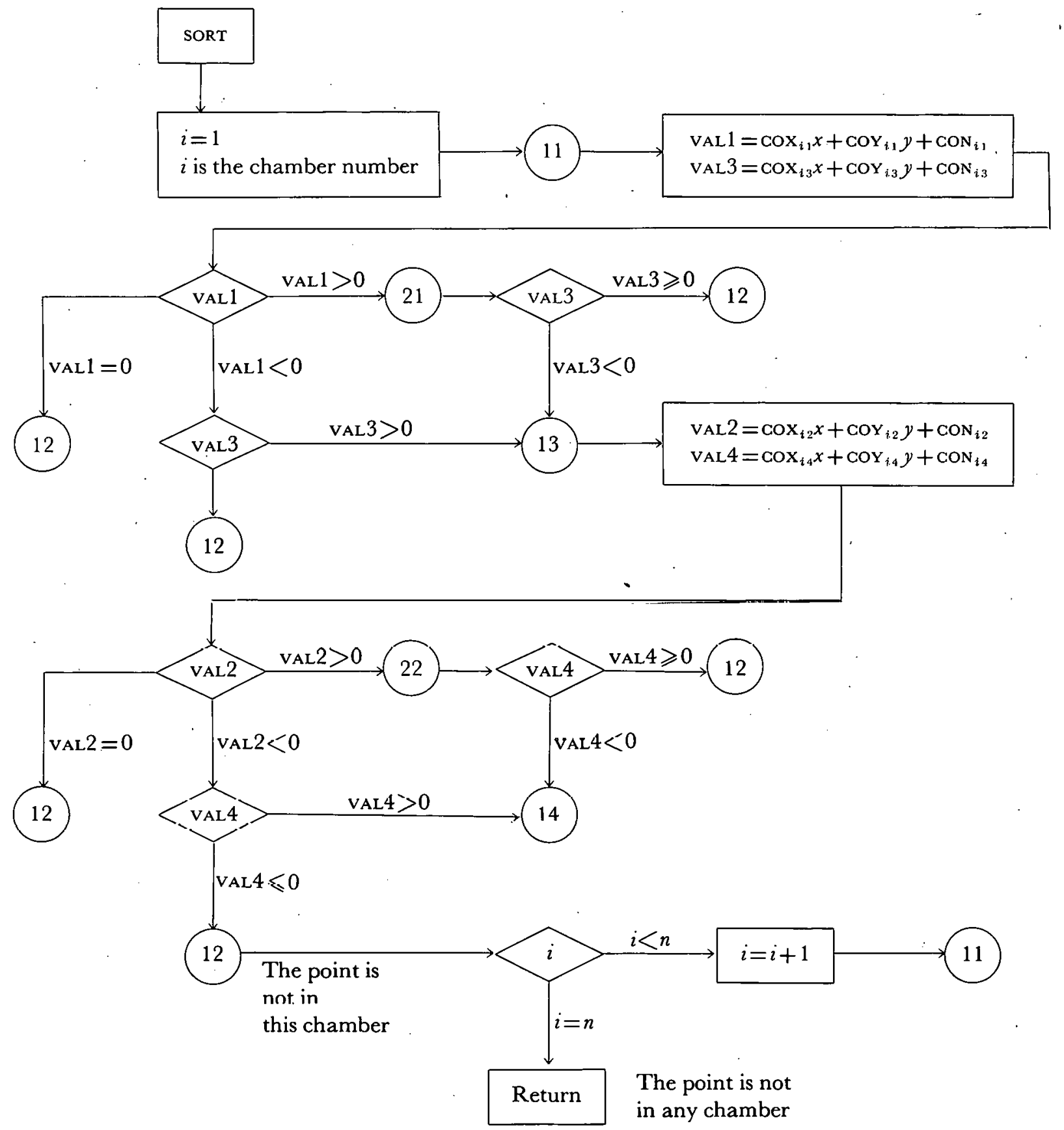



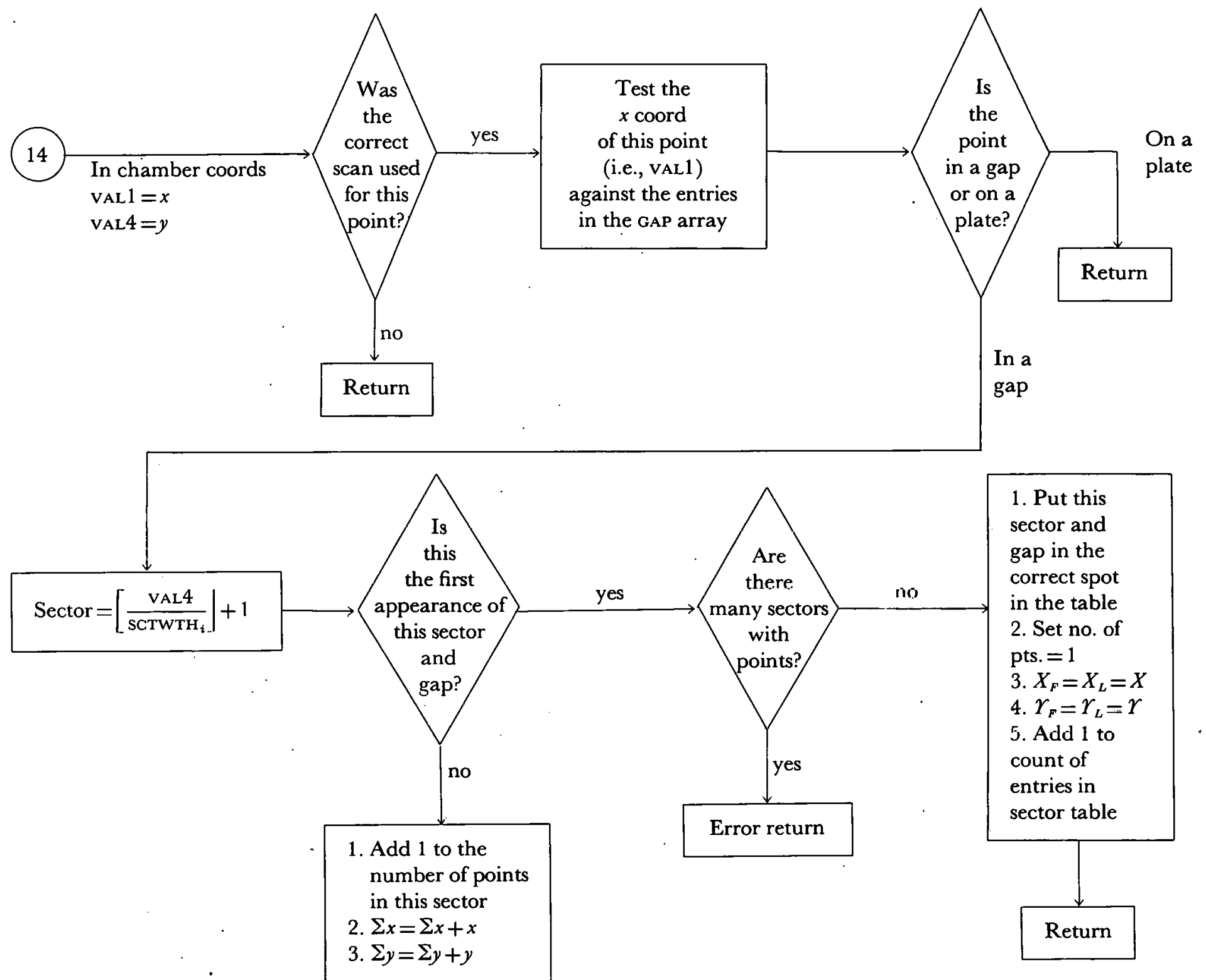


\section{Name: TRACK}

Purpose: Recognition of sparks and tracks; piecing together of segments of each track, including wide-angle tracks; least squares calculations of the equations of such tracks; preparation for kinematical analysis.

Input: This consists solely of a breakup of gaps in the given chambers into sectors. The actual input is in the form of a gapwise list of sectors with information in them, telling the number of points in the sector, and the $x$ and $y$ coordinates of the first point (smallest $x$ coordinate), last point (largest $x$ coordinate), and the centroid of points in the sector. The main portion of the input will actually be provided as input parameters, such as the number of chambers, number of gaps in each, etc. The nature of the input parameters will be clarified later in the write-up.

Description: The program itself can be broken up into several main parts, namely spark recognition, recognition of tracks nearly normal to the chamber plates, piecing together of segments of these, recognition of wide-angle tracks, piecing of segments of these, and final output.

The program starts essentially with spark recognition after a small amount of preparatory coding. This consists of dividing up large blocks of storage into smaller blucks, corresponding to different chambers. For example, if there are 2 chambers, ear.h with 20 gaps and 4 sparks per gap, 160 loca tions will be set aside for an array of $x$ coordinates of first and last points of sparks. The program will set aside the first 80 for chamber 1 and the sesnnd 80 for rhamher ?, and then list the addreoveo of the first words of the two blocks. After all storage is thus divided and records kept, the spark recognition starts.

The spark recognition is an analysis of the table of information by sectors, as stated in the sestion on input. The size of each sector is such that (a) no spark can cross two sector lincs (i.c., lic in three sectors), and (b) no two sparks can lie in one sector. The general procedure is to check the list of sectors represented in a given gap. Starting from the smallest sector number, check whether the first entry in this gap is followed by an entry from the next sector. If not, check whether the sector represented in the first entry has an acceptable number of points (currently, the limits are 2 and 15, but these are input parameters). If it has an acceptable number of points it is considered to be a spark, and its first and last $x$ coordinates are put in an array called XCRD 1 . Its first and last $y$ coordinates, as well as the $x$ and $y$ coordinates of its centroid, are put in array $\mathrm{CH} 1$. If the number of points is not acceptable, i.e., the next sector is not occupied, the entry is rejected as a spark.

The orun of the opark reeognition lics in lle allalysis uf cunseculive sectors with points. First, the sum of the number of points in the two sectors must be found. If this is below the lower limit, the ser.tor with the smaller sector number cannot contain a spark or part of one. The latter sector could contain part of a spark and is then checked in the same manner. If the sum is greater than the maximum number of points on a spark, the smaller sector number is tested alone, as if the next sector number were not represented. If the sum of points is within limits, the two spark segments are then chcckcd to see if they are close enough to form a spark. Four cases are checked as follows: (a) the last point in the former sector is compared with the first point in the latter sector, i.e., $\Delta y$ is compared with spsGD2 (Spark Segment Difference), an input parameter. If it is less than SPSGD2, we consider the two a spark with the new first and last points determined, the centroid computed, and the spark stored in the two arrays XCRDl and $\mathrm{CH}$ l; (b) if this case fails, the first point of the former and the last point of the latter are compared; similarly (c) the first points of each and (d) the last points of each are compared if all previous cases fail. If all four cases fail, the two sectors do not share a spark, and the former will be analyzed as if its sector number plus 1 were not represented. Thus if sectors $3,4,5$ in gap 11 contained data, there could be as many as 3 or as few as 0 sparks in those three sectors. At the end of the spark recognition the arrays of $x$ and $y$ coordinates will be complete.

Spark recognition is followed by the regular track recognition part of TRACK. TRACK analyzes mostly $y$ coordinates, with the $x$ 's utilized only in least squares fits. The ideal track consists of sparks in consecutive gaps within a small distance of each other in terms of $y$ coordinates ( $x$ coordinates 
vary with gap numbers). In general, any given spark will be matched with sparks in the next gap to see if there are any within a given distance of the former spark. This process does not always run smoothly, however.

Starting a track is the most complicated part of the process. A track starter is a spark which represents the smallest gap number (and sector number within any gap) of those sparks which have not yet been placed on tracks. When such a spark is found, the next gap is then analyzed to determine how many sparks are within a small distance $\mathrm{MU}$ (an input parameter) on either side of the track starter, and how many are not, but are within another distance DELTA on either side. The sparks thus found are divided up into those whose $y$ (first) coordinate ( $y$ coordinate of the point with smallest $x$ coordinate) is smaller than $y$ (last) of the track starter (minus sense), and those whose $y$ (first) is larger (plus sense). If a sense for this track has been established already, the total number of sparks within DELTA on that side and within a slight distance $M U$ on the other side (this allows for statistical jittering) is examined. If the number of sparks is 1, the spark is put on the track and takes over the role of the former spark, i.e., the sparks in the next gap are compared with it. If the number is 0 , this is considered a skipped gap and the count of such is increased by one. Once this count has reached NSKPS, currently 3 , the track is terminated and stored if it contains at least three sparks. At any stage of track recognition, once a spark is added to a track, the count of gaps skipped is started again. If there are two or more sparks within DELTA on the side of specified sense, a reference to them is set aside in a special array. Upon termination of the track, the sparks are substituted in the track equation until one is found which fits within FTLNE, an input parameter. When one fits, it is then put on the track, and the coefficients of the track are recomputed. A minimum of three sparks (other than multiple sparks) constitutes a track.

Starting a track when no sense is established is the hardest part. When a track starter is chosen and the next gap(s) examined, the sparks which fit within DELTA on minus and plus senses and those within MU form three classes. Analyzing the different cases involved forms the crux of the problem. The sum of all three classes is examined. If this is zero, the gap is considered a misfire or skip, and the next gap is considered, with the skip count increased and tested. At any time, a track will be terminated if this reaches NSKPS. If the total number which fit is one, there is no problem starting a track. If the spark is on the plus (minus) side, a track with plus (minus) sense is sought. If the spark is within $\mathrm{MU}$, i.e., in effect in the "equal" sense, no sense is established, as the track could later go either way. If the total number is two with one plus, and one minus, a track with minus sense is tried first. The plus spark is set aside to be tried when the other track is terminated. TRACK allows for track starters to start possibly two tracks, as in a "vee." If both sparks are on one side, say plus, plus sense will be established, the double spark will be stored, and the track continues in the plus sense. If one spark is in the "equal" and another on the plus (minus) side, this will be treated as if it were a double plus (minus) spark. If the total number of sparks that fit is three or more, the only case which will be considered is one on one side, two on the other. If any are "equal," the gap is skipped, avoiding triple sparks or ambiguities in sense. With one on one side, that sense will be established, and the double spark on the other side temporarily ignored. If there are at least two sparks in either sense which fit, the gap is skipped. Once there are three sparks on a track, DELTA will be modified. The absolute differences between the $y$ (last) of the first (and then the second) spark, and the $y$ (first) of the second (then the third) sparks are found, and divided by the differences in gap numbers. The maximum of these is taken, multiplied by $9 / 8$ (this can be changed), and $M U$ is added. The resulting number is a representative DELTA for this track. The DEL 1 array is replaced by multiples of the new number. After the track is temininated, the DEL 1 array is rcstored from the permanent record of it kept in the DELRG array.

Other facts should be noted. First, it should be pointed out that once a spark is on a track, it is never considered for another, with the exception of a track starter. When a track sense is established, since the track starter might be shared, it is stored separately. The other track will be in the other sense, and the next sense is stored. This can be used only once, however, so that once a track starter has been used twice to try to start a track, it is never used again. 
There is an option for the user as to whether two spark tracks will be considered. If so, a 2 is placed in the correct part of array NSPTK. When a track is terminated, NSPTK is examined if three sparks weren't found. If two were on the track, and two spark tracks are allowed in this chamber, the track will then be stored.

After the track recognition is completed, track segments are pieced together in the event that part of a track is missing in the middle. In order for two tracks to be pieced together, they must fulfill the following criteria: (a) one track must end in an earlier gap than that in which the other begins (i.e., no overlap); (b) the slopes of the two must be close enough (this depends on SLPDF, an input parameter); (c) the latter track, when extended back towards the former track, must pass very close to the last spark on the former track. This is accomplished by considering the equation of the latter track, $A X+B Y+C C=0$, and substituting the $x$ coordinate of the centroid of the last spark on the former track. The $y$ value thus found is subtracted from the $y$ coordinate of the centroid of said spark, and the absolute value of the difference is compared with ISCTD, an input parameter. If it is less than ISCTD, the two tracks are considered parts of one and are thus marked. Once the former track is matchcd with onc which starts later, it is not matched again, thus avoiding duplication of $\ddot{g} a \bar{p} \dot{s}$ in the final track. For example, if segment 1 starts in gap $a$ and cnds in gap $b$, wc would avoid matching both segment 2 from gaps $b+i$ to $b+j$, and segment 3 from gaps $b+k$ to $b+m$ with 1 , unless $k>j$. Thus the segments are put into equivalence classes representing the final tracks.

After the regular tracks are matched, the search for wide-angle tracks begins. A wide-angle track is one which forms a large angle with a line perpendicular to the plates, i.e., one which would not be found in the regular track recognition. A wide-angle track has the further stipulation that the sparks must pair off, and that if the first pair starts in an even (odd) gap, every pair on the track must start in an even (odd) gap. Only the sparks left after regular track recognition are considered for wide-angle tracks.

Wide-angle track recognition starts with the gap of smallest number containing an as yet unused spark. The next gap is then examined until an unused spark is found within distance WDDEL (an input parameter) from the former spark, constituting a pair. If none such is found, the first spark is rejected and another is sought. If such a spark is found, the next gap is examined for unused sparks, and the gap after that one is examined to complete the second pair. If the two gaps do not contain such a pair, the next two gaps are examined. Once three consecutive pairs of gaps fail to contain a pair of sparks as specified, the "track" is terminated, If it contains at least three pairs of sparks, the track is stored permanently. When two pairs of sparks are found, successive pairs of gaps are searched for more pairs. If three successive pairs of gaps fail to produce a pair of sparks which fits on the line already established, the track is terminated. Once a pair of sparks is found, a representative point is taken, namely the centroid of the two centroids. This point is substituted in the equation of the line through the other representative points and the resulting number is compared with DLWFT (Delta for wide fit, an input parameter). If the pair fits, the new equation of the track is computed including its representative point. If not, a new pair is sought. The method of terminating such a track is mentioned above. $\Lambda$ track with onc or two pairs is not stored. Wide-angle tracks are stored in the same manner as regular tracks. Each track is represented by the number of pairs of sparks in it, the coefficients of the track, and a list of sparks by gap number and the location of the spark in the CH1 array. Once all wide-angle track segments are found, no distinction is made between them and regular tracks.

At this stage, all track segments, regular and wide-angle, have been found and pieced together. What is left is the preparation for final output. The final stage of the program first goes through the list of regular tracks picking out all segments marked with a given number (same equivalence class). The sparks represented are combined as one track and the equation of the total track is then computed. As an option, a list of final tracks with their coefficients and a list of sparks can be printed out. This list consists of seven columns, the first two containing the $x$ and $y$ coordinates of the first point, then those of the last point and the centroids, with the seventh column containing the gap number of the spark. The same information is placed in a final array which is to be used as input 
to the view-matching program or kinematics, whatever the case may be. This final array contains a word telling the chamber number and the number of tracks in it. The tracks are then listed, each with a word denoting how many sparks it contains, and a list of the seven items (in four machine words) for each spark. This process is repeated for wide-angle tracks. Finally, the $\mathrm{CH} 1$ array is checked for sparks which are not on tracks, and a list of these is given as printed output under the heading of RESIDUE. The next file on the input data tape is read while control passes to the PRMCH and VEE rOutines (q.v.). After the execution of these two routines, the information equivalent to that contained in the print-out is written on an output tape by means of the FNOTPT routine. Control is then transferred back to TRACK and then to SORT and finally back to the Main Program.

Operating Insiructions and Input Parameters: TRACK is a general program, depending upon input parameters for operation on a specific experiment. These are assembly parameters, and a new assembly is necessary when any are changed. They are expected to remain constant in the analysis of all film from the same experiment. The parameters are as follows:

NCH (number of chambers). This parameter will be assembled with an EQU pseudo-operation, e.g., NCH EQU 3, as will those that follow.

MXPTS (the maximum number of points on a spark).

MNPTS (the minimum number of points on a spark).

MXGPS (the maximum number of gaps in any chamber).

NSKPS (the number of consecutive skipped gaps required to terminate a track; this number is currently 3$)$.

NGPS (number of gaps). This parameter is actually listed as an array of the number of gaps in each chamber, listed in chamber number order. For example, if there are 3 chambers with 48,24, and 8 gaps, respectively, NGrs would assemble as DEC 48,24,8.

NSPTK (number of sparks per track) is an array of 2's and 3's. If 2 spark tracks are allowed, a 2 is put in the array. If not, 3 is put in the array.

MXTRX and MNTRX are separate arrays of the maximum and minimum numbers of tracks allowed in the chambers, listed in the same manner as NGPS.

SPXPG is an array of the number of sparks per gap to allow in storage. If any gap has more than enough information for the allowed storage, information may be lost. The numbers in SPXPG should be large enough so that this does not happen.

RALMT is an array of numbers putting limits on the number of sparks being placed in the portions of the SPK1 array. Details on RALMT appear with SPK1. RALMT and SPXPG are similar to NGPS in nature.

Other parameters have to be determined after the experiment.

DEL 1 is an array listing delta, $2 *$ delta, $3^{*}$ delta, etc., where delta is the number used in regular track recognition going from one gap to the next. $2 *$ delta is used when the gap numbers differ by 2 , etc. The size of this array is equal to NSKPS.

DELRG is a permanent array identical to DEL1.

MU is a similar array of the distance between sparks to consider them "equal," i.e., the latter spark could fit on a track of any slope.

MU entries need not be in a $1: 2: 3: \ldots$ ratio.

SPSGD2 is the criterion for piecing together spark segments. It is the limit on $\Delta y$.

ISCTD is the limit on the difference between the projected $y$ coordinate of the latter track and actual $y$ coordinate of the last spark on the former track, used in piecing track segments.

ST.PDF is the limit on the difference in slopes in order to consider two tracks parallel, during piecing. WDDEL is the maximum difference between the last $y$ of the former spark and the first $y$ of the latter spark, used in order to consider them a pair for a wide-angle track.

DLWFT is the closeness criterion for the representative point of a new pair to fit to a wide-angle track. FTLNE has the same value as DLWFT and is used in putting one of a multiple spark on a regular track. 


$\begin{array}{llll}\text { NCH } & \text { EQU } & 2 & \\ \text { SPXPG } & \text { DEC } & 4,5 & \\ \text { NGPS } & \text { DEC } & 40,30 & \\ \text { RALMT } & \text { DEC } & 157,147 & \\ & \text { COMMON } & 620 & 2 * 40 * 4+2 * 30 * 5=\mathcal{N} \\ \text { CH1 } & \text { COMMON } & 1 & \\ & \text { COMMON } & 310 & \mathcal{N} / 2 \\ \text { XCRD1 } & \text { COMMON } & 1 & \\ & \text { COMMON } & 390 & \mathcal{N} / 2+40 * 2 \\ \text { SPK1 } & \text { COMMON } & 1 & \\ \text { USLSS } & \text { COMMON } & 3677 & (8000-\text { total so far }) \text { useless storage } \\ \text { BLOCK } & \text { COMMON } & 1 & .\end{array}$

Figute 14.

The remainder of the input parameters consists of storage areas whose sizes depend on the actual experiment, and are determined at assembly time. These areas are presently in common, and will continue so unless their total size exceeds 8000 , in which case some would have to be placed in the program area. $\mathrm{CH} 1$ is an array containing two words for every spark. The first word contains $y$ (first) and $y$ (last), and the second contains $y$ (centroid), $x$ (centroid), and the number of points in the spark. The array is divided up into a section for each chamber. Each section has its size equal to $2^{*}$ number of gaps * number of sparks per gap. Whether or not the maximum number of sparks per gap is reached, places are set aside for the maximum. Thus many will contain zeros. $\mathrm{cH} 1$ is assembled as COMMON $\mathcal{N}$ followed by CH 1 COMMON 1 , since $\mathrm{CH} 1$ is a forward-stored array. $\mathcal{N}=$ the sum of $2{ }^{*} \mathrm{NGPS}(I){ }^{*} \operatorname{SPXPG}(I)$ where $I$ goes from 1 to $\mathrm{NCH}$.

XCRD 1 is an array of $x$ coordinates and requires 1 word per spark. It is assembled as COMMON $N / 2$, followed by XCRD 1 COMMON 1 , where $\mathcal{N}$ is computed as per CH 1 .

SPK1 is an array of references to track segments. Each track reference contains $n+4$ words for a track of $n$ sparks. Three words are set aside for the cocfficicnts of the track equation, and one for the number of sparks.

SPK 1 is assembled as COMMON $\mathcal{N} / 2+40^{*} \mathrm{NCH}$, followed by SPK 1 COMMON 1 . Forty places are thus set aside for possibly 10 segments. In order to make sure that none of these arrays overflows, RALMT is an array of limits on each section of the SPK 1 array. Entries in RALMT are stored in increasing chamber number order and will be slightly less than the amount of storage in SPK1 for each chamber, so that the addition of one more track would not overflow the bounds. The size of the three arrays, SPK1, CH 1, and XCRDL 1, should actually be computed before assembly and not put as formulas listed here.

The total of the sizes of all common storage thus allocated should be subtractcd from 8000 , and an area of useless common should follow the references to those just mentioned. This is followed by BLOCK COMMON 1.

Example: Consider 2 chambers, 40 gaps in the first, and 30 in the second, with a maximum of 4 and 5 sparks per gap, respectively. The assembly would contain the parameters shown in Figure 14 .

Length: Approximately 2700 executable instructions.

Time: Approximately $0.3 \mathrm{sec}$ for 3000 data points in a file.

Date: June 1965.

Author: M. Strongson. 
Name: VeE

Purpose: Determination as to whether two given tracks form a V, i.e., whether their intersection is near two of their endpoints.

Input: Input consists of the locations of blocks of information concerning each of the tracks. Each block which is output from TRACK will consist of the number of sparks on the track, a list of the coefficients of its least squares line, and a list of the coordinates of representative points on each spark in the track.

Description: The equations of the two tracks can be considered as

$$
A(I) * X+(B(I) * Y+C(I)=0(I=1,2) .
$$

The intersection of the tracks is found by Cramer's rule, i.e., the intersection point will be

$$
\begin{aligned}
& \left\{\left[B(1)^{*} C(2)-C(1)^{*} B(2)\right] /\left[A(1) * B(2)-B(1)^{*} A(2)\right],\right. \\
& \left.\left[C(1)^{*} A(2)-A(1)^{*} C(2)\right] /\left[A(1)^{*} B(2)-B(1)^{*} A(2)\right]\right\},
\end{aligned}
$$

if the tracks are not too close to being parallel.

The tracks are considered too close to being parallel if the denominator in either of the expressions in the above ordered pair is smaller than its respective numerator, or is 0 .

Once the intersection point is determined, it is then compared with the endpoints (four in all) of the two tracks involved. If the distances between the vertex and one endpoint from each track are less than or equal to the input parameter DLMT, the tracks are said to form a $\mathrm{V}$. The locations of the two successful endpoints are given as return input to the main program. An analysis program can then determine if the vee is of a type allowed (sometimes only a $\mathrm{V}$ whose vertex is close to the first sparks of both tracks might be desired, for example).

VEE is called by a calling sequence of four words, which are in the following order:

$\begin{array}{lll}\text { TSX } & \text { VEE, } 4 & \\ \text { PZE } & \text { A } & \text { Location of first track } \\ \text { PZE } & \text { B } & \text { Location of second track } \\ \text { PZE } & 0 & \text { To be replaced by the } x \text { coordinate of the vertex }\end{array}$

The $x$ coordinate is returned to give an indication as to which gap contains the actual intersection of the two tracks. All three arguments of the calling sequence must be positive.

Length: 100 locations.

Timø: Approximately $1 \mathrm{msec}$ per pair of tracks.

Date: June 1965.

Author: M. Strongson.

\section{Name: PRMCH}

Purpose: Two tracks (A and B) in one chamber are matched with two tracks ( $C$ and $D)$ in another view of that chamber. The purpose of PRMCH is to determine which of the following four possibilities exists: (1) A matches with $\mathrm{C}$, and $\mathrm{B}$ with $\mathrm{D}$; (2) A matches with $\mathrm{D}$, and B with $\mathrm{C}$; (3) both of the above possibilities occur at once (error; ambiguity); (4) no good match is found. [PRMCH can also determine whether or not a match exists between two tracks (one-to-one case)].

Method: The following two requirements must be satisfied if two tracks are to match. (For definiteness, call the two tracks $\mathrm{A}$ and C.) $R 1$ : If track $\mathrm{A}$ begins in gap $x$ and ends in gap $y$, then track $\mathrm{C}$ must begin in one of the gaps $x$ - Param $1, x-($ Param $1-1), \ldots, x, x+1, x+2, \ldots, x+$ Param 1 (PARAM1 is a parameter, currently $=1$ ) and end in one of the gaps $y$-PARAM1, $\ldots, y+$ PARAM1. $R 2$ : Track A cannot disagree in more than PARAM2 gaps, where PARAM2 is a parameter, currently $=3$. A disagreement in a gap refers to a condition where one track has a spark in the gap and the other one doesn't. 


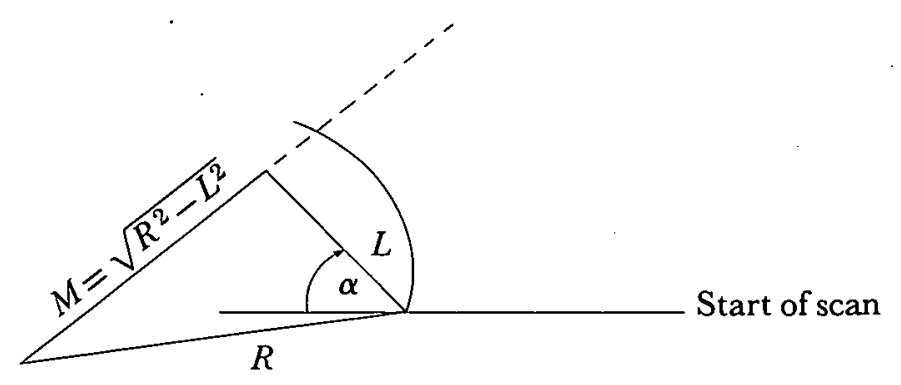

Figure 15.

(Internal subroutines are used to determine whether or not these requirements hold for a pair of tracks.)

If $R 1$ and $R 2$ are not sufficient to cstablish a dcfinitc match (that is, if both possiblc pairs meet both requirements), then the following criteria will be used:

If $A$ and $C$ have fewer disagreements than $A$ and $D$, we will tentatively say that $A$ matches with C. Then, if $B$ and $D$ have fewer disagreements than $B$ and $C$, we will say that $A$ matches with $C$ and $B$ with $D$. If, however, $B$ and $C$ have fewer disagreements than $B$ and $D$, we will call the result an ambiguity.

NoTE: DIS $(A, C)$ or DISAC refers to the number of disagreements between $A$ and $C$. These hold for all other possibilities.

Length: 360 executable instructions.

Error Refurns: (1) If there is no match found, the ERROR program is called to write "NO MATCH IN GENERAL CASE" (2-2 case) or "NO MATCH IN 1-1 CASE." Return is made to the calling program after the message is written and the first argument to PRMCH (see below) is set to +0. (2) If there is an ambiguity, the ERROR program is called to write "AMBIGUITY IN MATCHING PROGRAM." The first argument is now set to -0 before return is made.

Requirements: There will be four arguments to $\mathrm{PRMCH}$. They will be leading words for track arrays which will have, in their addresses, the number of sparks in the track (If $B=0$, a 1-1 match is desired.)

Also, TARAM1 and rARAM2 are required; these have been explaincd previously.

If there is a match (say A-C $\mid B-D$ ), the arguments, which are given to PRMCH as ABCD (this order), will be put back in the order ACBD:

Date: May 1965.

Authors: J. Malanient, L. Padwa.

\section{Name: VARY}

Purpose: Calibration of the digitizer, by finding the correct values of $R, L$, and $\alpha$. (See Figure 15.)

Method: VARY is a FORTRAN program which systematically varies the values of $R, L$, and $\alpha$. The three parameters may be varied independently of each other, and for fixed $R$ and $\alpha$, different $L$ 's may be used for the two different scans.

For each set of $R, L$, and $\alpha$, a simple computation for $M$ is performed (see Figure 15), and then the fiducial program is called three times to find three fiducials whose correct positions are known.

In transforming the fiducials, the current values of $R, M, L$, and $\alpha$ are used.

VARY then prints, for each fiducial triple, the coordinates of the fiducials and the distances between them. Since the correct distances are known quantities, the user can select the values of $R, L$, and $\alpha$ which yield distances closest to the correct ones. 
To avoid going through much unnecessary output (i.e., where the distances differ greatly from the correct ones) an option exists whereby printing is suppressed except for a narrow range of distances centered about the correct distance. Other simple options are available to the user who has a knowledge of FORTRAN.

The ranges of variation and the increments in $R, L$, and $\alpha$ are up to the user. In general, any change in variation requires a recompilation of VARY.

Date: August 1965.

Authors: L. Padwa, S. Heller.

\section{Name: CRCFT}

Purpose: CRCFT is a subroutine designed to fit a circle through a set of points and list the deviation of each point from the best fit circle. This routine can be used to verify the standard deviation.

Method: A preliminary examination of the data points (by looking at a dump of rectangular coordinates of the data) determines an approximate radius and center. Only points falling within a set tolerance of this guessed circle are used in the fit. If the points are $\left(x_{i}, y_{i}\right)$ and the equation of the "best tit" circle is

$$
R^{2}=(x-A)^{2}+(y-B)^{2},
$$

the function $D$ minimized is

$$
D=\sum_{i}\left\{R^{2}-\left[\left(x_{i}-A\right)^{2}+\left(y_{i}-B\right)^{2}\right]\right\}^{2},
$$

i.e., the square of the difference of the found radius squared and the best fit radius squared. Upon setting

$$
\frac{\partial D}{\partial R}=\frac{\partial D}{\partial A}=\frac{\partial D}{\partial B}=0
$$

and solving,

$$
\begin{aligned}
& A=1 / 2 \frac{\left(\overline{x^{3}}-\bar{x} \overline{x^{2}}+\overline{x y^{2}}-\bar{x} \overline{y^{2}}\right)\left(\overline{y^{2}}-\bar{y}^{2}\right)-\left(\overline{y^{3}}-\bar{y} \overline{y^{2}}+\overline{y x^{2}}-\bar{y} \overline{x^{2}}\right)(\overline{x y}-\bar{x} \bar{y})}{\left(\overline{x^{2}}-\bar{x}^{2}\right)\left(\overline{y^{2}}-\bar{y}^{2}\right)-(\overline{x y}-\overline{x y})^{2}} \\
& B=1 / 2 \frac{\left.\left(\overline{y^{3}}-\bar{y} \overline{y^{2}}+\overline{y x^{2}}-\bar{y} \overline{x^{2}}\right)\left(\overline{x^{2}}-\bar{x}^{2}\right)-\overline{x^{3}}-\bar{x} \overline{x^{2}}+\overline{x y^{2}}-\bar{x} \overline{y^{2}}\right)(\overline{x y}-\bar{x} \bar{y})}{\left(\overline{x^{2}}-\bar{x}^{2}\right)\left(\overline{y^{2}}-\bar{y}^{2}\right)-(\overline{x y}-\bar{x} \bar{y})^{2}}
\end{aligned}
$$

and

$$
R=\overline{x^{2}}+\overline{y^{2}}-2 \bar{x} A-2 \bar{y} B+A^{\dot{2}}+B^{2}
$$

where

$$
\overline{\alpha^{j}}=\frac{1}{n} \sum_{i=1}^{n}\left(\alpha_{i}\right)^{j}
$$

Input: One card is read in with the FORTRAN Format 4F14.8 having the numbers $x$ center, $y$ center, radius, and tolerance on the radius (all in digitizer $x$ units).

Output: An equation of the circle, and each point with its deviation from the radius $R$.

Use: CRCFT is called by a FAP program called MAIN whose purpose it is to read the magnetic tape and transfer the rectangular coordinates to the subroutine CRCFT.

Date: August 1965.

Author: S. Heller. 


\section{REFERENCES}

1. G. Schwender, Report on the Logical Description of the Spark Chamber Reader, BNL 877 (T-353), June 1964.

2. S. Heller, L. Padwa, and I. Perlman, STEAM: A Computer Program To Analyze Spark Chamber Data Obtained by Use of an Automatic Flying Spot Digitizer, BNL 833 (T-237), Dec. 1963.

3. S. Heller and G. Schwender, Report on a Spark Chamber Reader System, BNL 969 (T-407), Nov. 1965.

4. G. Schwender, D. Engelberg, R. Schuman, and R. SQuires, Report on the Logical Description of the Film Preselector for the Spark Chamber Reader, BNL 876 (T-352), June 1964.

\section{ACKNOWLEDGMENT}

The programmers would like to thank the many people from the Applied Mathematics and Physics Departments at Brookhaven National Laboratory who aided in the development of the Spark Chamber Reader system. 